\title{
DETECTING VASCULAR-TARGETING EFFECTS OF THE HYPOXIC CYTOTOXIN TIRAPAZAMINE IN TUMOR XENOGRAFTS USING MAGNETIC RESONANCE IMAGING
}

\author{
Lauren J. Bains, M.Sc., * Jennifer H. E. Baker, B.Sc., ${ }^{\dagger}$ Alastair H. Kyle, Ph.D., ${ }^{\dagger}$ \\ Andrew I. Minchinton, Ph.D., ${ }^{\dagger}$ and Stefan A. Reinsberg, Ph.D.* \\ * Department of Physics and Astronomy, University of British Columbia, Vancouver, Canada; and ${ }^{\dagger}$ Medical Biophysics Department, \\ British Columbia Cancer Research Centre, Vancouver, Canada
}

\begin{abstract}
Purpose: To determine whether vascular-targeting effects can be detected in vivo using magnetic resonance imaging (MRI).

Methods and Materials: MR images of HCT-116 xenograft-bearing mice were acquired at 7 Tesla before and 24 hours after intraperitoneal injections of tirapazamine. Quantitative dynamic contrast-enhanced MRI analyses were performed to evaluate changes in tumor perfusion using two biomarkers: the volume transfer constant $\left(K^{\text {trans }}\right)$ and the initial area under the concentration-time curve (IAUC). We used novel implanted fiducial markers to obtain cryosections that corresponded to MR image planes from excised tumors; quantitative immunohistochemical mapping of tumor vasculature, perfusion, and necrosis enabled correlative analysis between these and MR images. Results: Conventional histological analysis showed lower vascular perfusion or greater amounts of necrosis in the central regions of five of eight tirapazamine-treated tumors, with three treated tumors showing no vascular dysfunction response. MRI data reflected this result, and a striking decrease in both $K^{\text {trans }}$ and IAUC values was seen with the responsive tumors. Retrospective evaluation of pretreatment MRI parameters revealed that those tumors that did not respond to the vascular-targeting effects of tirapazamine had significantly higher pretreatment $K^{\text {trans }}$ and IAUC values.

Conclusions: MRI-derived parameter maps showed good agreement with histological tumor mapping. MRI was found to be an effective tool for noninvasively monitoring and predicting tirapazamine-mediated central vascular dysfunction. (C) 2009 Elsevier Inc.
\end{abstract}

Hypoxic cytotoxins, Dynamic contrast-enhanced MRI, Vascular shutdown, Vascular dysfunction, Immunohistochemistry, Tumor mapping, Fiducial marker.

\section{INTRODUCTION}

The presence of hypoxic cells within tumors reduces the effectiveness of radiotherapy, and is a known mechanism for tumor radioresistance (1). One strategy to combat this resistance is to utilize bioreductive cytotoxins, prodrugs that are reduced under low-oxygen conditions to cytotoxic metabolites. This selective toxicity to hypoxic cells would complement the preferential toxicity of radiotherapy for oxygenated tumor cells. Tirapazamine (3-amino1,2,4-benzotriazine-1,4-di- $N$-oxide; SR 259075 [formerly SR 4233]) is the most advanced hypoxic cytotoxin under clinical development (for a comprehensive review of tirapazamine, see reference 2).
Tirapazamine (TPZ) is reduced under hypoxic conditions to an oxidizing species that reacts with DNA to cause double-strand breaks (3). In vivo experiments have shown anticancer activity of TPZ in multiple tumor models and in combination with radiotherapy $(4,5)$. However, the anticancer activity of TPZ is a paradox, as compelling evidence using spheroids and multicellular cultures suggests that TPZ is unable to penetrate efficiently through tumor tissue to reach chronically hypoxic cells located far from vasculature (6-9). This poor penetration of TPZ has been attributed to bioreductive inactivation at intermediate oxygen tensions more proximal to vasculature $(9,10)$. We have previously reported an observation of the unexpected ability of TPZ
Reprint requests to: Stefan A. Reinsberg, Ph.D., 6224 Agricultural Rd., Department of Physics and Astronomy, University of British Columbia, Vancouver V6T 1Z1, Canada. Tel: (604) 822-2925; Fax: (604) 822-5324; E-mail: reinsberg@physics.ubc.ca

Lauren J. Bains and Jennifer H. E. Baker contributed equally to this article.

Supported by CIHR. J. H. E. B. and A. H. K. are recipients of the MSFHR senior graduate studentship.
Conflict of interest: none.

Acknowledgment-We thank Piotr Kozlowski and Andrew Yung for MR imaging and Lynsey Huxham for synthesizing tirapazamine and assisting with preliminary mouse studies.

Received April 21, 2008, and in revised form Nov 18, 2008. Accepted for publication Nov 20, 2008. 
to mediate central vascular dysfunction in murine syngeneic and human xenograft tumors grown in mice $(11,12)$. This mechanism for activity of TPZ could have a significant impact on its application if found to be clinically relevant.

Monitoring drug effects on tumor blood volume, flow, and permeability noninvasively can be accomplished using magnetic resonance imaging (MRI). MRI has been used extensively in both preclinical and clinical situations to investigate the effects of vasculature-targeting agents such as combretastatin (13), N-acetylcolchinol-O-phosphate (14), and 5,6-dimethylxanthenone-4-acetic acid (15). The current study presents a method for evaluating the effects of TPZ noninvasively, using two dynamic contrast-enhanced MRI (DCE-MRI)-derived biomarkers for the assessment of antivascular agents: initial area under the curve (IAUC) and volume transfer constant $\left(\mathrm{K}^{\text {trans }}\right)$. HCT-116 tumor-bearing mice received baseline MR scans, were subsequently treated with TPZ, and received a follow-up scan. Cryosections were then mapped for vasculature, perfusion, and necrosis using histological tumor mapping techniques in order to confirm observations with DCE-MRI and to validate the fact that the vascular-damaging effects of TPZ are observable using conventional DCE-MRI, a technique that is in current clinical use. DCE-MRI has the potential to be highly beneficial for determining whether TPZ-mediated loss of perfusion occurs in patients and whether this is a mechanism that contributes to its clinical anticancer activity.

\section{METHODS AND MATERIALS}

\section{Mice, fiducial markers, and tumors}

NOD/SCID mice 8 to 11 weeks old, weighing 19 to $24 \mathrm{~g}$, were bred in our institutional animal facility; experiments in the study were approved by the Animal Care Committee of the University of British Columbia. Fiducial markers were constructed of polyethylene tubing (inner diameter, $0.58 \mathrm{~mm}$ ) and filled with paraffin wax and saline, creating an MR-visible interface. Marker tubes were implanted subcutaneously in the sacral region of mice, in a craniocaudal orientation, 2 days prior to subcutaneous implantation of HCT-116 cells. Histological sections and MRI slices were taken so that the fiducial marker lay perpendicular to the imaging plane of each slice; the fixed nature of the marker ensured small angular differences between sectioning planes for histological and serial MR image slices. The mean weight \pm standard error of the mean (SEM) of excised tumors was 512 $\pm 68 \mathrm{mg}$.

\section{Treatments}

Mice were anesthetized with isoflurane, received an MRI scan and were administered either TPZ or vehicle 1 hour after removal from anesthesia. TPZ (synthesized by L.A. Huxham [11]) was administered by intraperitoneal injection at $60 \mathrm{mg} / \mathrm{kg}(0.34 \mathrm{mmol} /$ $\mathrm{kg}$ ), using a $1.25 \mathrm{mg} / \mathrm{ml}$ solution in saline. A second MRI scan was obtained $24 \mathrm{hrs}$ after treatment; mice awakened promptly following anesthesia, however, some TPZ-treated mice required longer periods in which to recover to their preanesthesia fitness state. No correlation was found between length of recovery time and response to TPZ treatment. Mice that recovered within $30 \mathrm{~min}$ postanesthesia received intraperitoneal doses of $500 \mathrm{mg} / \mathrm{kg} 5$ bromo-2-deoxyuridine (BrdUrd) (Sigma Chemical, Oakville, ON, Canada) as a back-up perfusion marker (16), with tumor excision at 1 hour post-BrdUrd administration. All mice received a $35-\mu \mathrm{l}$ intravenous dose of $0.6 \mathrm{mg} / \mathrm{ml}$ carbocyanine (DioC7; Molecular Probes, Eugene, OR) in 75\% dimethylsulfoxide 5 minutes before euthanasia. Excised tumors were embedded and frozen with fiducial markers at $-20^{\circ} \mathrm{C}$.

\section{MRI}

MR images were acquired using a $7 \mathrm{~T}$ horizontal bore Bruker BioSpec 70/30 scanner with a transmit/receive solenoid coil. A fast low-angle shot (FLASH) sequence (6 slices, 1-mm thickness, 0.5$\mathrm{mm}$ gap, $312.5-\mu \mathrm{m}$ in-plane resolution) with a repetition time (TR) of $226 \mathrm{~ms}$ was performed, followed by a FLASH with a TR of $113 \mathrm{~ms}$; both sequences used a flip angle of $75^{\circ}$. A contrast agent (30 mM gadolinium [Gd]-diethylenetriamine penta-acetic acid, 10 $\mu \mathrm{l} / \mathrm{g}$ ) was injected via a tail vein catheter at $18 \mu \mathrm{l} / \mathrm{sec}$ using a power injector. Ten precontrast and 124 postcontrast image stacks were acquired with a time resolution of $14.5 \mathrm{~s}$.

\section{Immunohistochemical staining and image acquisition}

Serial-step $10-\mu \mathrm{m}$ cryosections were cut at 0.5 -mm intervals, imaged for carbocyanine fluorescence, and fixed in acetone-methanol for $10 \mathrm{~min}$. Vasculature and proliferation were stained using antibodies for CD31 and incorporated BrdUrd, respectively (17), and slides were counterstained with hematoxylin. Whole-tumor sections were imaged as previously described (18) using a system which allowed for tiling of adjacent microscope fields of view such that images of entire tumor cryosections were captured at a resolution of 1.5 $\mu \mathrm{m} /$ pixel.

\section{Image analysis}

Similar image analysis methods have been previously reported, with additional detail provided below $(11,12)$. Using both fiducial and anatomical landmarks, we chose six cryosections per tumor to match the MRI slices. Using ImageJ- and user-supplied algorithms, digital images were superimposed (19). Tumors were manually cropped to tumor tissue boundaries; staining artifacts and necrosis were subsequently cropped from hematoxylin-stained images. The necrotic fraction (NF) was calculated as the ratio of necrotic pixels to total pixels. Positive fluorescence for CD31 and carbocyanine images was obtained by applying a threshold ( $>5$ standard deviations [SDs] above background), with neighboring positive pixels grouped as "objects." The perfused vessel fraction (PF) was calculated as the proportion of CD31-positive objects that were also at minimum 20\% overlapped with positive carbocyanine pixels on the overlaid image. Data for individual tumors are displayed as mean \pm SEM values for six analyzed slices per tumor.

Each histological section was manually aligned with the corresponding MR slice, using customized Interactive Data Language (IDL; ITT) software. For MRI/histological image correlative analyses, a threshold was determined for carbocyanine images with a pixel size of $1.5 \mu \mathrm{m} \times 1.5 \mu \mathrm{m}$, and the number of carbocyanine-positive objects in an area corresponding to an MRI pixel $(0.3125 \mathrm{~mm} \times$ $0.3125 \mathrm{~mm}$ ) was calculated. A $0.3 \times 0.3 \mathrm{~mm}$ resolution "carbocyanine map" depicting pixels at intensities representative of the number of perfused vessels in the histological sections was produced. 


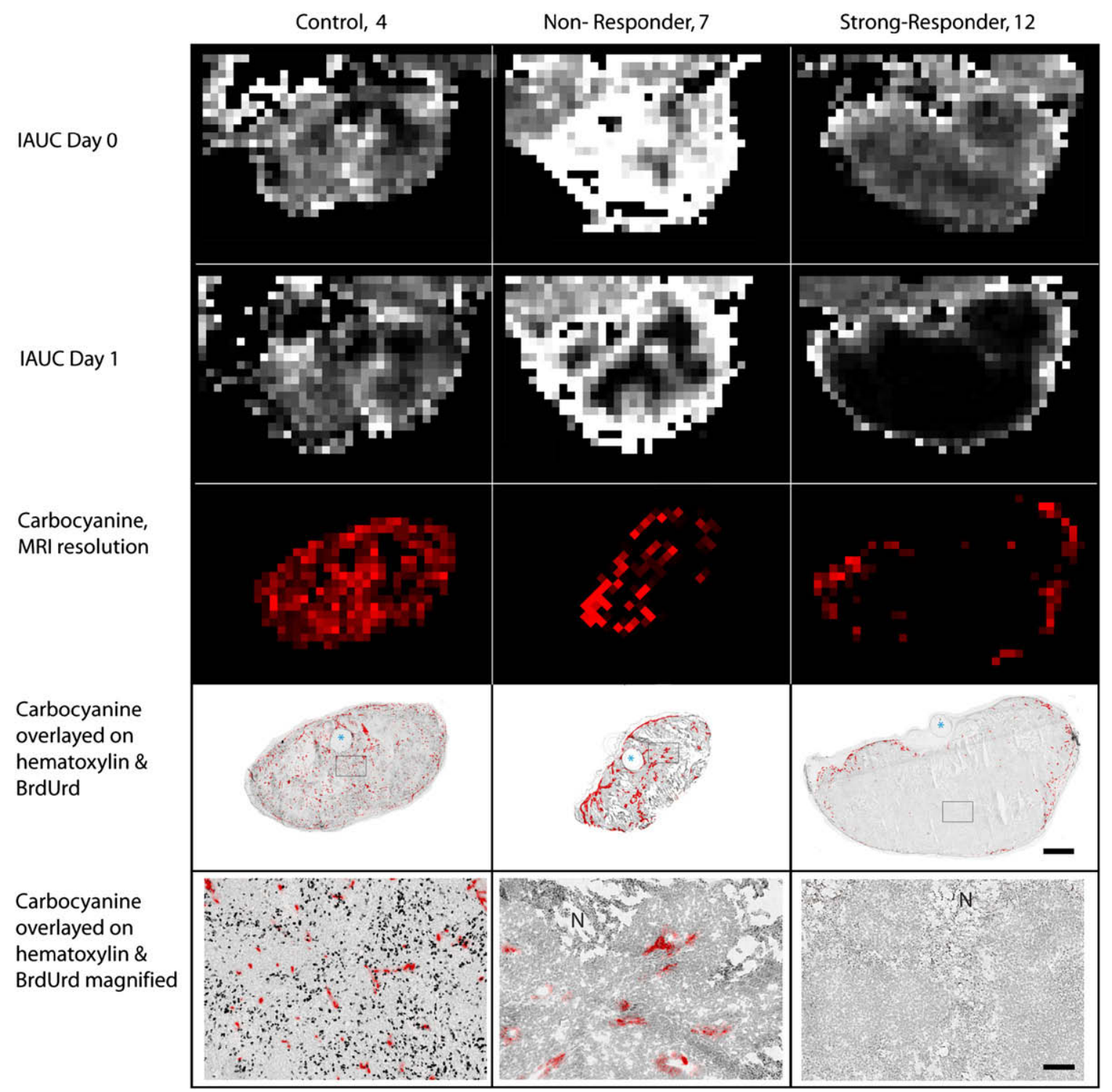

Fig. 1. DCE-MRI and histological parameter maps of HCT-116 tumors. IAUC for baseline day 0 (row 1 ) and day 1 (row 2). Parameter maps showing the number of perfused vessels measured using histology, displayed at MR resolution (row 3). Histological tumor maps are shown as composite images of carbocyanine fluorescence (red) overlaid on the hematoxylin counter-stained tissue background (grey), with BrdUrd staining in black (row 4). Boxed areas indicate magnified regions (row 5); $\mathrm{N}=$ necrotic regions; blue stars = placement of fiducial markers. Row 4 scale bar, $1250 \mu \mathrm{m} ;$ row $5,125 \mu \mathrm{m}$.

\section{Scoring vascular dysfunction response}

A combined vascular dysfunction score (VDS) was calculated from NF and PF relative to that of control (PFC) parameters, using the formula

$$
\mathrm{VDS}=1-(1-\mathrm{NF}) \times \mathrm{PFC}
$$

The PFC was calculated as the proportion of perfused vessels for a tumor divided by the maximum proportion of perfused vessels observed in controls. The VDS will be 0 if there is no NF (i.e., $\mathrm{NF}=0$ ) and normal perfusion $(\mathrm{PFC}=1)$. The VDS will be higher in cases of increased necrosis or reduced perfusion. This objective score permits quantitative evaluation of tumors that may have a loss of perfusion measurable using either the CD31 staining combined with a perfusion marker or the necrosis in tu- mors that have no remaining CD31. Tumors with a VDS of $>2$ SDs higher than the mean control score are considered responsive to TPZ.

\section{DCE-MRI analysis}

Customized IDL software was used to calculate changes in $\mathrm{T}_{1}$, using signal intensity images from preinjection $(\mathrm{TR}=226 \mathrm{~ms})$ and baseline $(\mathrm{TR}=113 \mathrm{~ms})$ scans to create concentration time curves. Relaxivity of the contrast agent was determined with a phantom study using diluted contrast agent samples. IAUC values were calculated by integrating the concentration time curves from the beginning of enhancement to the fourth frame (60 s postcontrast arrival). A two-compartment Kety model was used (20) to find the $\mathrm{K}^{\text {trans }}$, a parameter describing the movement of contrast agent from blood plasma into the extravascular extracellular space, and $\mathrm{v}_{\mathrm{e}}$, the fraction of extravascular extracellular space (21). The relationship between the 




Fig. 2. DCE-MRI and histological parameter maps of HCT-116 tumors. Details are as described in the legend to Fig. 1 and in text.

concentrations of contrast agent in the tissue and in the blood plasma are described by the equation,

$$
C_{t}(t)=K^{\text {trans }} \int_{0}^{t} C_{p}(\tau) e^{\frac{-K^{\text {trans }}}{v_{e}}(t-\tau)} d \tau
$$

where $C_{t}(t)$ and $C_{p}(t)$ are the time-dependent concentration of contrast agent in the tissue and vascular plasma, respectively. Data for individual tumors are displayed as the means \pm SEM for all six analyzed slices per tumor.

\section{Radial analysis}

Customized radial intensity analysis IDL software was created to quantify each parameter as a function of distance from the tumor edge. Starting from the outside edge, $312.5 \mu \mathrm{m}$ rims were created, and parameter means were pooled for all six slices from a tumor. As a measure of tumor radial heterogeneity, the percent difference was found between two weighted means: the outer one-third and the inner two-thirds of the tumor.

\section{Statistics}

All statistical analyses were performed using GraphPad Prism software (version 4.0c for Macintosh). Nonparametric tests were used in all instances: typically a Kruskal-Wallis analysis of variance rank test, followed by Dunn's posttests between groups. The $p$ values of $<0.05$ and $<0.01$ are reported. A paired Wilcoxon rank test was performed for matched sample analyses. Spearman's rank correlation was used for IAUC mean values, and Pearson's correlation coefficient with a $95 \%$ confidence interval is reported for regression analyses for IAUC of radial profiles vs. carbocyanine fluorescence.

\section{RESULTS}

HCT-116 tumor-bearing mice were examined using DCE-MRI before and after treatment with TPZ, and immunohistochemistry-based tumor mapping was subsequently performed. Figure 1 shows images of both MRI and tumor 

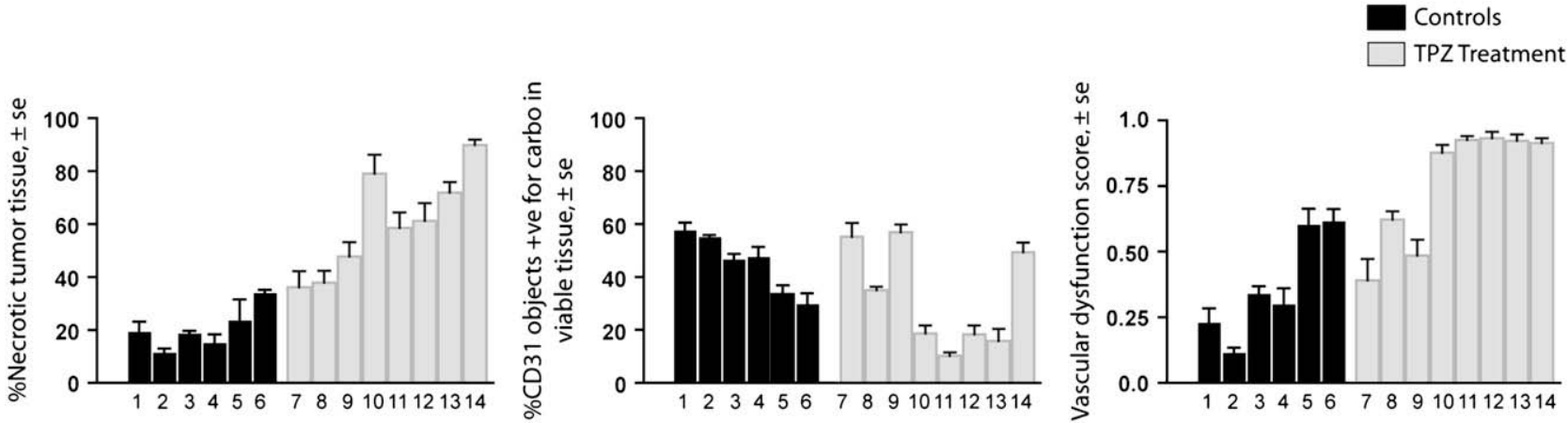

Fig. 3. Histological analysis showing amounts of (A) necrosis and (B) perfusion in sections of control and tirapazaminetreated tumors. (C) Combined VDS incorporating both necrosis and perfusion values. Bars represent means for individual tumors.

mapping modalities and displays sample tumors from untreated control (tumor 4) and TPZ-treated mice that either did (tumor 12) or did not (tumor 7) respond with central vascular dysfunction following TPZ treatment. Figure 2 illustrates further examples of TPZ-treated tumors, including one tumor with no response (tumor 9) and two tumors with strong evidence of central vascular dysfunction (tumors 13 and 14).

\section{A) Mean IAUC}

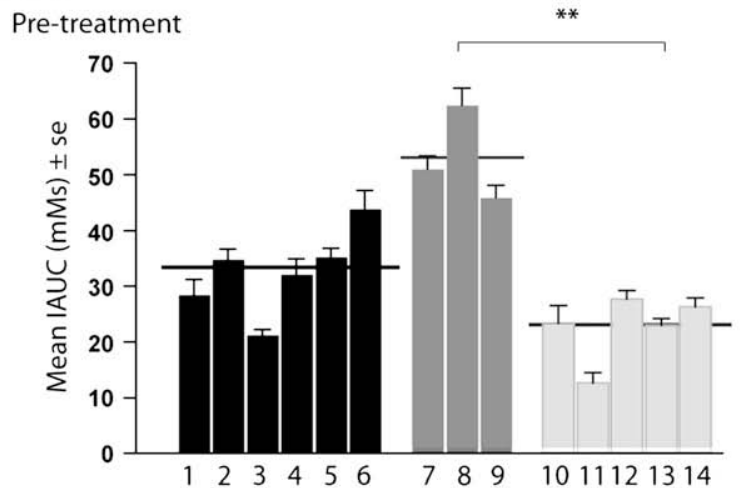

B) Mean $\mathrm{K}^{\text {trans }}$

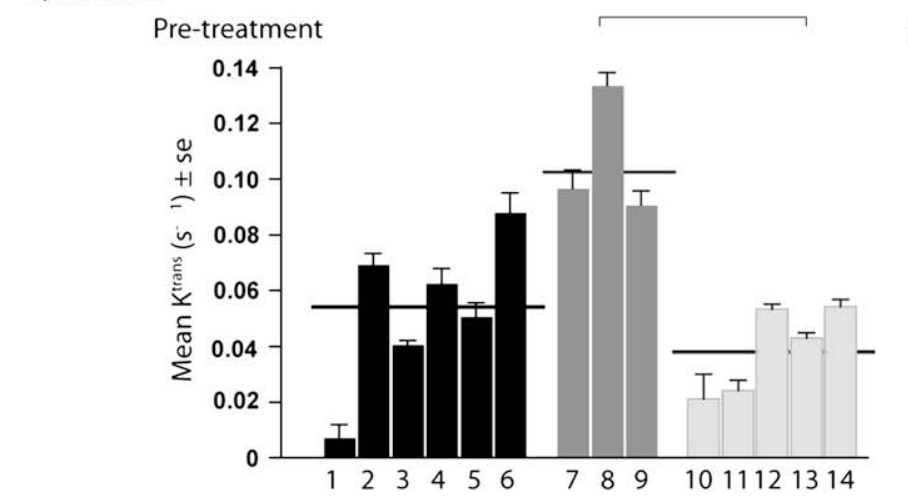

Histological analysis identified tumors with an increase in necrosis or a decrease in perfusion in response to TPZ

Response to TPZ was assessed by examining both the number of perfused vessels (Fig. 3B) and the amount of necrosis (Fig. 3A). Combined VDSs were calculated for all tumors (Fig. 3C), with controls showing a combined vascular dysfunction scores (VDS) mean \pm SD of $0.386 \pm 0.203$. Tumors 7, 8, and 9 (Fig. 3C) each had VDSs that were within 2
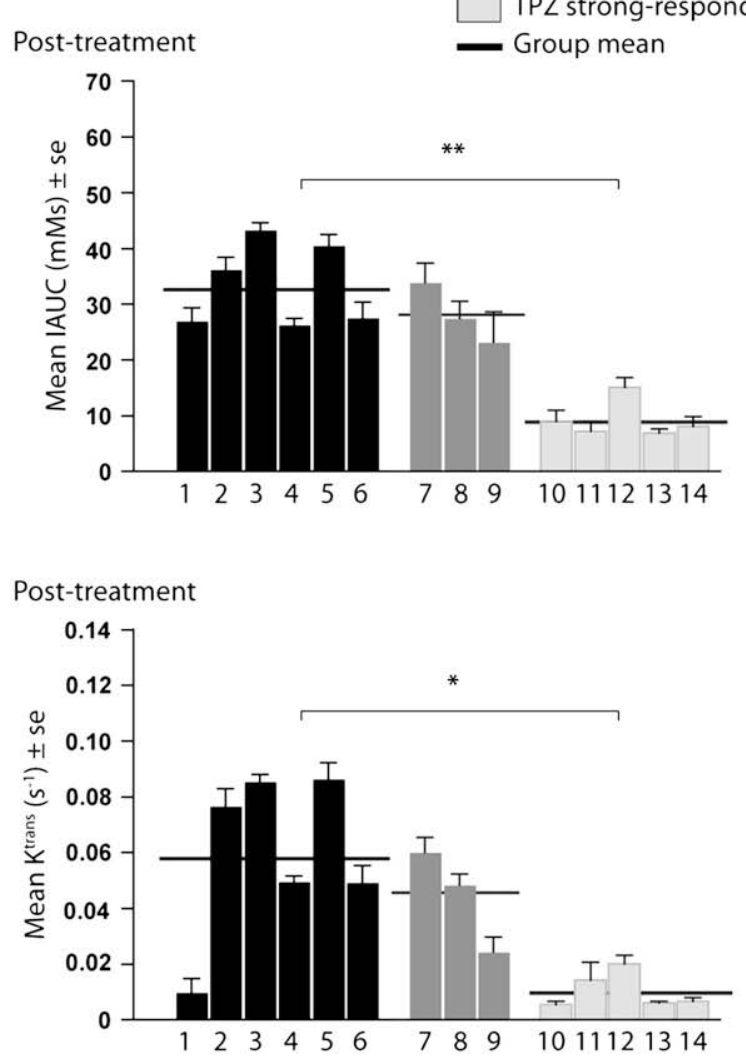

Fig. 4. Tirapazamine-mediated changes in vascular function measured using the DCE-MRI parameters IAUC and $\mathrm{K}^{\text {trans }}$; bars represent individual tumors, while horizontal lines indicate group means. TPZ-treated groups retrospectively classed as non- and strong responders based on histological data $(*, p<0.05)(* *, p<0.01)$. 

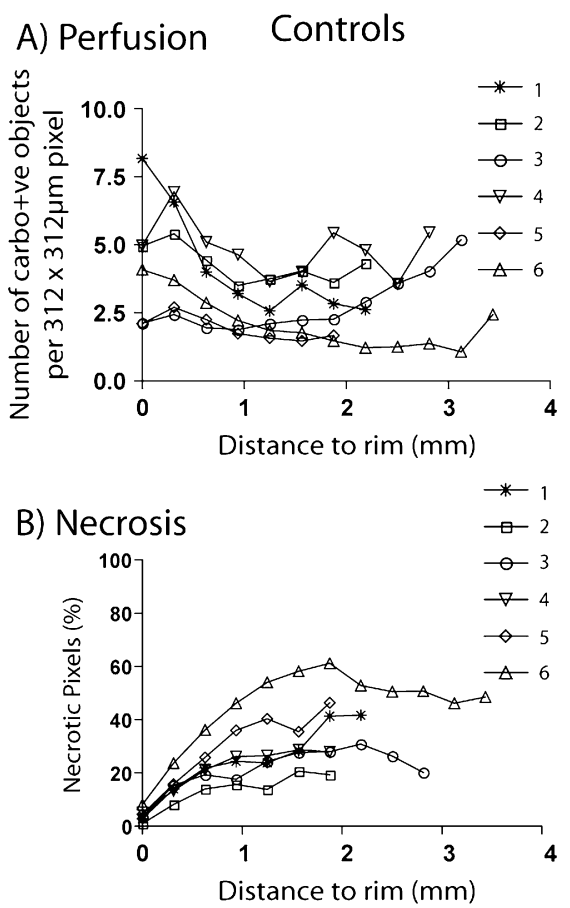

Non-Responders
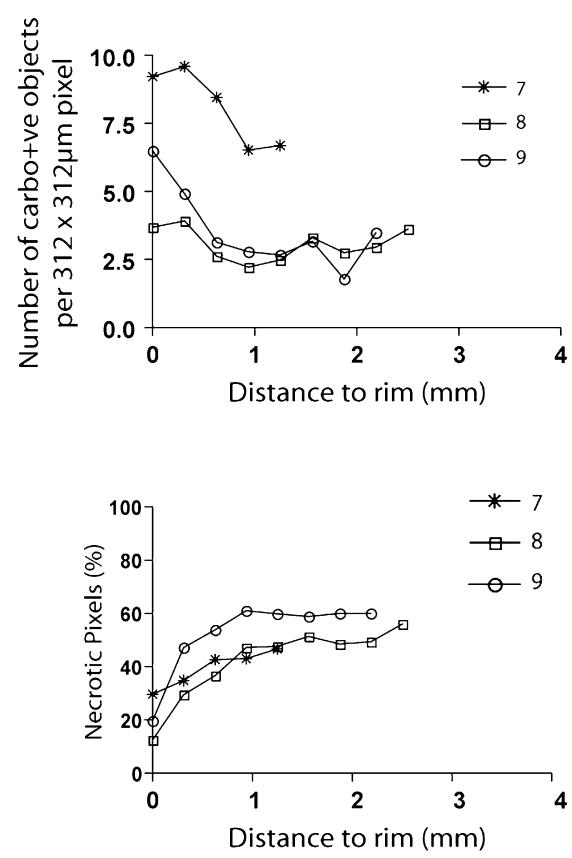

Strong-Responders
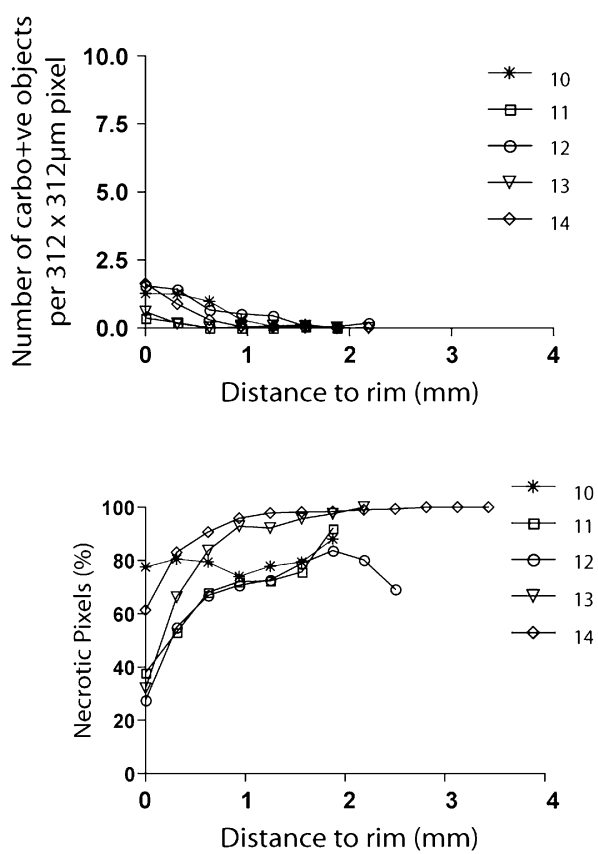

Fig. 5. Tumour mapping microregional heterogeneity in response to TPZ. Radial analyses of images show large amounts of necrosis, and small amounts of perfusion are seen predominantly in the central regions of TPZ-treated tumors.

SDs of controls and were subsequently classed as "nonresponders," whereas tumors 10 to 14 were more than 2 SDs higher than control values and were objectively classified as "responders." This proportion of responder and nonresponder HCT-116 tumors treated with TPZ is consistent with our previous studies examining vascular dysfunction response using tumor mapping data (11). Analysis of incorporated BrdUrd is not included due to the adequacy of carbocyanine for labeling of perfused vasculature and an insufficient number of mice being administered the marker for analysis of proliferation.

\section{DCE-MRI analysis shows decreases in IAUC and $K^{\text {trans }}$ values for TPZ-treated tumors with central vascular dysfunction}

Control tumors show no significant changes in their preand posttreatment IAUC values, while TPZ-treated tumors show a combined average reduction of 52\% $(p<0.05)$. Whole-tumor mean IAUC values for all tumors are shown in Fig. 4, with retrospective classification of TPZ-treated tumors into non- and strong-responder categories based on histological data (VDS). Strongly responding tumors have significantly lower posttreatment IAUC values relative to controls $(p<0.01)$, whereas nonresponders maintain values similar to controls. Nonresponders also exhibit elevated mean pretreatment IAUC values relative to strong responders $(p<0.01)$; pretreatment IAUC and vascular dysfunction scores in response to TPZ treatment are significantly correlated using the Spearman's rank test $(\rho=-0.667$; one-sided, $p<0.05$ ). Spearman's rank correlation is significant between pre- and posttreatment IAUC values for all treated tumors, in- dicating that a higher pretreatment IAUC value predicts for higher posttreatment IAUC $(\rho=0.929$; one-sided, $p<$ 0.01 ). Note that prior to the retrospective separation of tumors into non- and strong responders, the mean baseline IAUC value for the treated group was indistinguishable from that of the control group.

Mean $\mathrm{K}^{\text {trans }}$ values exhibit similar trends. Strong responders show marked reduction of average $\mathrm{K}^{\text {trans }}(73 \%$ reduction; $p<0.01$ ), while nonresponders show a nonsignificant $(p=0.1)$ average loss compared to pretreatment values (59\% reduction). As shown in Fig. 4B, elevated pretreatment $\mathrm{K}^{\text {trans }}$ values are seen in those tumors that did not respond to TPZ treatment relative to those of the strong responders $(p<$ 0.01 ), and only the strong responders have a significantly lower posttreatment mean $\mathrm{K}^{\text {trans }}$ value than controls $(p<$ $0.05)$.

\section{Microregional heterogeneity of response to TPZ}

MRI and histological maps (Fig. 1 and 2) illustrate the fact that treated tumors experienced a loss of perfusion in central regions but retained a peripheral rim of perfused tissue. Figure 5A shows the average number of carbocyanine-positive vessels as a function of distance from the tumor margin. Control (Fig. 5A, left panel) and nonresponders (Fig. 5A, middle panel) show comparable amounts of carbocyanine throughout their tissues, compared to strong responders (Fig. 5A, right panel) with only a few perfused vessels remaining at the rim. Figure 5B shows the radial distribution of necrotic tissue; as is typical for HCT-116 xenografts, necrosis is observed in treatment-naive tumors (Fig. 5B, left panel), particularly in the central regions. This necrosis is 
Controls
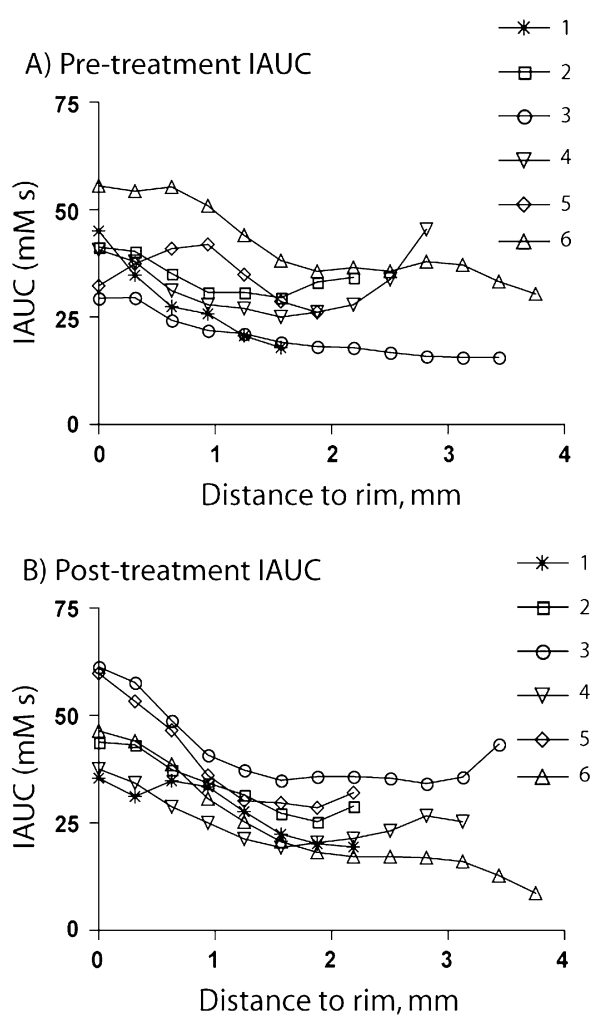

Non-Responders

Strong-Responders
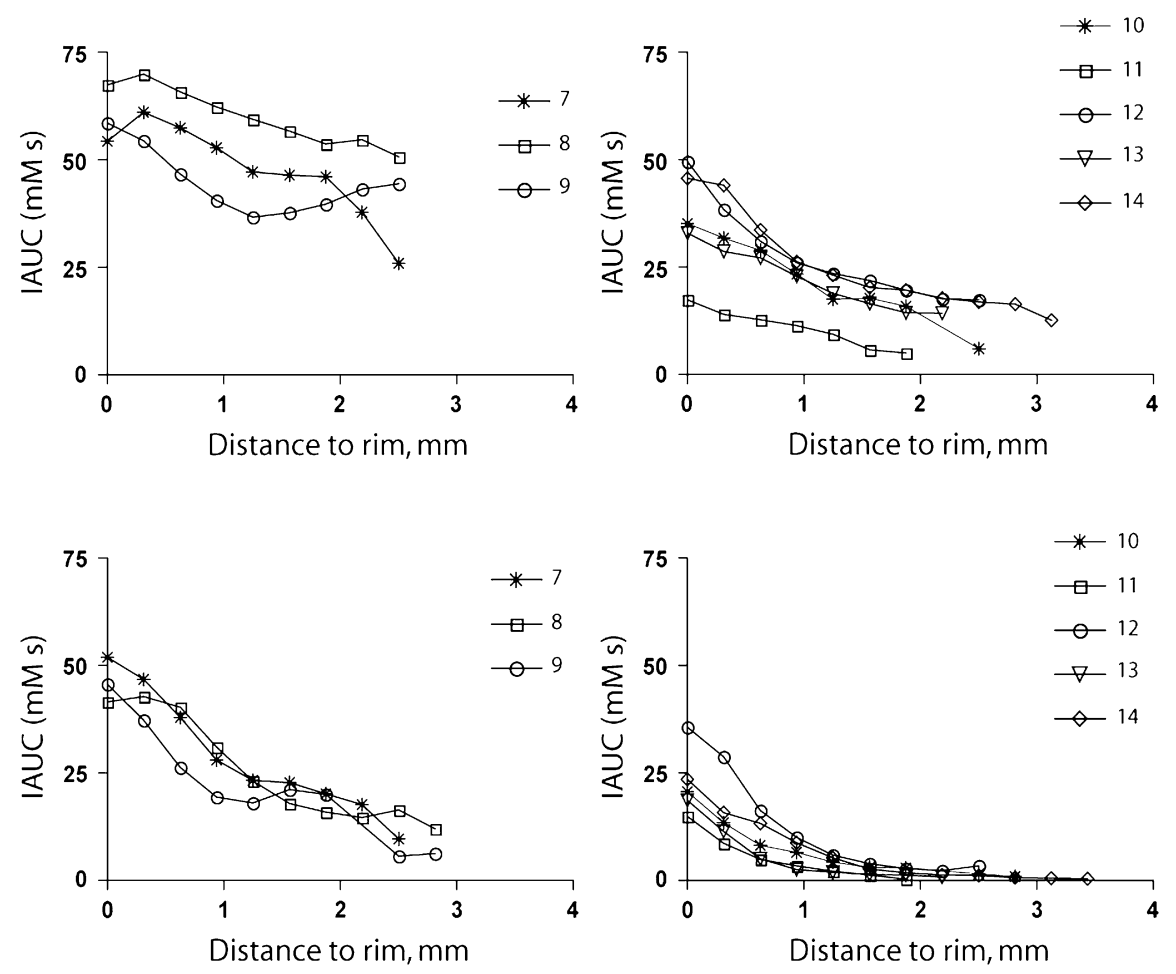

Fig. 6. MRI microregional heterogeneity in response to tirapazamine. Losses in perfusion occur predominantly in the central regions of tumors, as measured by using radial analyses of either IAUC or $\mathrm{K}^{\text {trans }}$ parameter maps.

elevated slightly for nonresponders (Fig. 5B, middle panel) and is increased dramatically in strong responders (Fig. 5B, right panel), reaching nearly $100 \%$ at a distance as close as $1 \mathrm{~mm}$ to the tumor margin.

Similar analysis for IAUC is shown in Fig. 6, where the radial distribution of IAUC in all tumors before treatment (Fig. 6A) exhibits a slight gradient, with higher values at the periphery relative to those at distances farther from the margin. No significant changes were observed in follow-up scans of control tumors (Fig. 6B, left panel). After treatment with TPZ, tumors classed as strong responders (Fig. 6B, right panel) show mean IAUC values approaching 0 toward the center of tumors, while nonresponders show IAUC values that are reduced compared to those of pretreatment levels but do not reach 0 (Fig. 6B, middle panel).

Radial distribution data are summarized by a constructed index of heterogeneity based on the ratio of mean rim (outer one-third) to center (inner two-third) values. For all measured parameters (IAUC, $\mathrm{K}^{\text {trans }}$, carbocyanine, and necrosis), the index of heterogeneity is greater for TPZ-treated tumors. A significant correlation was observed between IAUC and carbocyanine-based measurements of radial heterogeneity (Pearson correlation, $r=0.88$, with $95 \%$ confidence intervals, 0.57-0.97) using this index confirming that MRI and histological measurements show quantitative as well as qualitative agreement.

\section{DISCUSSION}

Although the effectiveness of TPZ has been attributed to its cytotoxicity to hypoxic cells, its poor tissue penetration profile (6-9) presents a paradox regarding its mechanisms for anticancer activity. Using immunohistochemistry-based tumor mapping, we have previously shown that TPZ has an unexpected vascular-targeting effect in multiple tumor models $(11,12)$, leading to the important question of whether this effect also occurs in patients.

Our study employed three major steps: a baseline DCEMRI scan followed by TPZ treatment, a follow-up scan at 24 hours after treatment, and finally, tumor excision and histological analysis. The visual correlations between the three measurements of perfusion are quite striking, particularly in light of the experimental difficulties inherent in such a comparison. The use of simple implanted fiducial markers (Fig. 1 and 2) greatly reduced variations in slice angulation and position that were observed in preliminary studies using a detachable external marker. This suggests that internal markers are a more reliable solution for longitudinal studies of tissue that moves as easily as a subcutaneous tumor.

With the assistance of these fiducial markers, we obtained tumor cryosections that closely approximated the MRI slices, and histological tumor maps for vascular perfusion and tumor necrosis were analyzed. In all of the tumor models reported in previous studies, the typical rate of response seen is 
approximately 6 to 7 responding out of 10 treated tumors, with the remaining tumors having little or no detectable vascular dysfunction effects measurable with the static tumor maps $(11,12)$. Tumors that respond to TPZ treatment exhibit an almost total loss of perfused vessels or significantly greater levels of necrosis in the central regions of tissue. Combined vascular dysfunction scores (VDS) provides an objective, quantitative measure of the vascular-targeting effects of TPZ, and identified tumors 7, 8, and 9 as nonresponders and the remaining tumors 10 to 14 as responders (Fig. 3C). It is necessary to consider both necrosis and perfusion, as the timing for onset of necrosis and subsequent destruction of unperfused vessels following central vascular dysfunction are somewhat variable, creating an experimental artifact where tissue that sustains loss of perfusion due to treatment but which has already become necrotic no longer exhibits CD31 staining and is therefore not reflected in the loss-of-perfusion calculations (Fig. 3).

BrdUrd is a useful proliferation marker that is delivered to tumor tissue through the vascular system, which means that it can function as a perfusion marker (16). However, in cases of vascular dysfunction, it is difficult to differentiate between poor delivery of the marker and a decrease in proliferation in areas of low BrdUrd incorporation. In our previous studies, low BrdUrd staining reflected a decrease in proliferation in areas around perfused blood vessels following TPZ administration, with large areas of unperfused tissue also showing a complete lack of BrdUrd incorporation, likely due to a combination of decreased proliferation and poor delivery of the marker $(11,12)$. In the current study, BrdUrd was administered at a lower dose to some mice to assist in the determination of large areas of perfusion loss using the tumor mapping images; however, carbocyanine was successfully injected intravenously in every animal, providing a more reliable marker for perfusion assessment. Our MR imaging protocol was unable to evaluate proliferation for useful comparisons between tumor mapping and imaging, making the proliferation data of relatively low value for this study and it is consequently not shown.

IAUC and $\mathrm{K}^{\text {trans }}$ are related measures of tumor perfusion, where IAUC is a semiquantitative measure of contrast agent delivery and $\mathrm{K}^{\text {trans }}$ is most often interpreted as a measure of blood flow and vascular permeability. The observed posttreatment decrease in both IAUC and $\mathrm{K}^{\text {trans }}$ values confirms that TPZ treatment can induce vascular changes to a degree that is measurable and quantifiable using MRI (Fig. 4).

MRI and histological parameter maps qualitatively depict vascular changes occurring preferentially in the central regions of tumors, and quantitative radial analyses confirmed these observations for IAUC, $\mathrm{K}^{\text {trans }}$, necrosis, and histologically assessed perfusion (Fig. 5 and 6). The heterogeneity index created by relating values from the peripheral to the central regions of tumors permitted a comparison between the two imaging modalities in this study; parameters from histological tumor mapping and MRI were found to have good correlation.
A significant advantage of using MRI is the ability to examine longitudinal data. When data from those tumors identified as nonresponders (tumors 7, 8, and 9) using our conventional tumor mapping are examined retrospectively, it can be seen that prior to TPZ treatment, these tumors exhibited greater IAUC and $\mathrm{K}^{\text {trans }}$ values than those of the rest of the treatment-naive tumors; a strong correlation was found between pretreatment IAUC and tumor response. Posttreatment MRI data show that the values for nonresponding tumors did decrease but not to the same degree as those seen in the strong responders; an overall positive correlation was found between pre- and posttreatment measurements for both the IAUC and the $\mathrm{K}^{\text {trans }}$.

These data suggest that tumors that are more likely to be sensitive to the vascular-targeting effects of TPZ may be identified prior to treatment as those which have low levels of perfusion. Although the sample size is small in this study, this observation is important, considering the hypoxic cytotoxic mechanism of TPZ, and suggests that the vascular-targeting mechanism of TPZ may be related to hypoxia. Support for a hypoxia-associated predictor for the clinical success of TPZ treatment comes from findings by Rischin et al. (22). They found that patients with hypoxic primary tumors as identified on ${ }^{18} \mathrm{~F}$-misonidazole positron emission tomography more often benefit from TPZ/cisplatin treatment than do patients with nonhypoxic primaries: 2 of 3 nonhypoxic patients failed locally, whereas only 1 of 19 hypoxic patients exhibited locoregional failure. No data were presented in their study to indicate whether the anticancer activity was a consequence of a vascular-targeting effect.

Gd-based contrast agents are in clinical use and would therefore be practical for determining whether a vascular-targeting mechanism for TPZ could be responsible for its clinical efficacy. The MRI protocol used in these experiments is directly transferable to the clinic, and the biomarkers for treatment response identified here should be further examined for their diagnostic utility. In particular, trials studying the efficacy of TPZ should be accompanied by DCE-MRI studies with sufficient time and spatial resolution to create IAUC maps and examine heterogeneities in tumor perfusion.

\section{CONCLUSIONS}

This study illustrates that tumor perfusion measured with IAUC and $\mathrm{K}^{\text {trans }}$ is a candidate imaging biomarker for tumor response to TPZ treatment in animal models, using a protocol that is transferable to humans. The use of novel implanted fiducial markers and analysis of microregional heterogeneity showed that MRI data correspond well with histological findings. Tumors sensitive to the vascular-targeting effects of TPZ sustained measurable losses in perfusion and onset of necrosis in the central regions of tissue. Those few tumors that did not respond were found to have exhibited significantly higher pretreatment perfusion values as measured by IAUC and $\mathrm{K}^{\text {trans }}$; this observation suggests an approach for further studies examining predictors for TPZ efficacy. 


\section{REFERENCES}

1. Gray L, Conger A, Ebert M, Hornsey S, Scott O. The concentration of oxygen dissolved in tissues at the time of irradiation as a factor in radiotherapy. Br J Radiol 1953;26: 638-648.

2. Marcu L, Olver I. Tirapazamine: from bench to clinical trials. Curr Clin Pharmacol 2006;1:71-79.

3. Anderson R, Shinde S, Hay M, Gamage S, Denny W. Activation of 3-amino1,2,4-benzotriazine 1,4-dioxide antitumor agents to oxidizing species following their one-electron reduction. J Am Chem Soc 2003;125:748-756.

4. Zeman E, Hirst V, Lemmon M, Brown J. Enhancement of radiation-induced tumor cell killing by the hypoxic cell toxin SR 4233. Radiother Oncol 1988;12:209-218.

5. Dorie M, Menke D, Brown J. Comparison of the enhancement of tumor responses to fractionated irradiation by SR 4233 (tirapazamine) and by nicotinamide with carbogen. Int $J$ Radiat Oncol Biol Phys 1994;28:145-150.

6. Durand R, Olive P. Evaluation of bioreductive drugs in multicell spheroids. Int J Radiat Oncol Biol Phys 1992;22: 689-692.

7. Durand R, Olive P. Physiologic and cytotoxic effects of tirapazamine in tumor-bearing mice. Radiat Oncol Investig 1997;5: 213-219.

8. Hicks K, Fleming Y, Siim B, Koch C, Wilson W. Extravascular diffusion of tirapazamine: Effect of metabolic consumption assessed using the multicellular layer model. Int J Radiat Oncol Biol Phys 1998;42:641-649.

9. Kyle A, Minchinton A. Measurement of delivery and metabolism of tirapazamine to tumour tissue using the multilayered cell culture model. Cancer Chemother Pharmacol 1999;43: 213-220.

10. Cardenas-Navia L, Secomb T, Dewhirst M. Effects of fluctuating oxygenation on tirapazamine efficacy: Theoretical predictions. Int J Radiat Oncol Biol Phys 2007;67: 581-586.

11. Huxham LA, Kyle AH, Baker JHE, McNicol KL, Minchinton AI. Tirapazamine causes vascular dysfunction in HCT-116 tumour xenografts. Radiother Oncol 2006;78:138-145.
12. Huxham L, Kyle A, Baker J, McNicol K, Minchinton A. Exploring vascular dysfunction caused by tirapazamine. Microvasc Res 2008;75:247-255.

13. Galbraith SM, Maxwell RJ, Lodge MA, et al. Combretastatin A4 phosphate has tumor antivascular activity in rat and man as demonstrated by dynamic magnetic resonance imaging. J Clin Oncol 2003;21:2831-2842.

14. Robinson SP, McIntyre DJO, Checkley D, et al. Tumour dose response to the antivascular agent ZD6126 assessed by magnetic resonance imaging. Br J Cancer 2003;88:1592-1597.

15. McPhail LD, McIntyre DJO, Ludwig C, et al. Rat tumor response to the vascular-disrupting agent 5,6-dimethylxanthenone-4-acetic acid as measured by DCE-MRI, plasma 5-hydroxyindoleacetic acid levels, and tumor necrosis. Neoplasia 2006;8:199-206.

16. Janssen H, Ljungkvist A, Rijken P, et al. Thymidine analogues to assess microperfusion in human tumors. Int J Radiat Oncol Biol Phys 2005;62:1169-1175.

17. Kyle A, Huxham L, Baker J, Burston H, Minchinton A. Tumor distribution of bromodeoxyuridine-labeled cells is strongly dose dependent. Cancer Res 2003;63:5707-5711.

18. Kyle A, Huxham L, Yeoman D, Minchinton A. Limited tissue penetration of taxanes: A mechanism for resistance in solid tumors. Clin Cancer Res 2007;13:2804-2810.

19. Abramoff MD, Magelhaes PJ, Ram SJ. Image processing with ImageJ. Biophotonics International 2004;11:36-42.

20. Tofts PS, Brix G, Buckley DL, et al. Estimating kinetic parameters from dynamic contrast-enhanced $\mathrm{T}_{1}$-weighted MRI of a diffusable tracer: standardized quantities and symbols. $J$ Magn Reson Imaging 1999;10:223-232.

21. Tofts PS, Kermode AG. Measurement of the blood-brain barrier permeability and leakage space using dynamic MR imaging. Magn Reson Med 1991;17:357-367.

22. Rischin D, Hicks RJ, Fisher R, et al. Prognostic significance of ${ }^{18}$ F-misonidazole PET-detected tumor hypoxia in patients with advanced head and neck cancer randomly assigned to chemoradiation with or without tirapazamine. J Clin Oncol 2006;24: 2098-2104. 